\title{
EPIDEMIOLOGICAL AND CLINICAL ASPECTS OF DERMATOPHYTOSES IN EASTERN SLOVAKIA: A RETROSPECTIVE THREE-YEAR STUDY
}

\author{
Zuzana Baranová', Tomáš Kampe ${ }^{2}$, Erik Dorko³ ${ }^{3}$ Kvetoslava Rimárová \\ 'Department of Dermatovenerology, Faculty of Medicine, Pavol Jozef Šafárik University in Košice, Slovak Republic \\ ${ }^{2}$ Department of Dermatovenerology, Louis Pasteur University Hospital, Košice, Slovak Republic \\ ${ }^{3}$ Department of Public Health and Hygiene, Faculty of Medicine, Pavol Jozef Šafárik University in Košice, Slovak Republic
}

\begin{abstract}
SUMMARY
Objective: A three-year retrospective study of fungi isolated from samples of patients with suspected fungal skin infections in Eastern Slovakia is presented.

Methods: A total of 11,989 samples were collected and investigated with direct microscopic examination using $20 \% \mathrm{KOH}$ and cultivated in Sabouraud and Mycosel medium. Identification was based on macroscopic and microscopic characteristics.

Results: Of the total samples, $61.76 \%(7,405 / 11,989)$ were completely negative and $38.24 \%$ positive $(4,584 / 11,989)$. Dermatophytes accounted for $45.88 \%$ of isolates $(2,103 / 4,584)$, yeasts for $26.79 \%$ (1,228/4,584), non-dermatophytes for $15.29 \%$ (701/4,584), and Malassezia sp. for $12.4 \%(552 / 4,584)$. Trichophyton rubrum was the most prevalent causative agent $(79.08 \%)$ implicated in fungal skin infections, followed by Trichophyton interdigitale (10.60\%). Less frequent isolates included Trichophyton tonsurans $(5.13 \%)$, and Trichophyton mentagrophytes (3.14\%). Other dermatophytes (Microsporum audouinii, Microsporum gypseum, Microsporum canis, Trichophyton violaceum, Trichophyton verrucosum, and Epidermophyton floccosum) were very rarely identified (each in less than $1 \%$ of all samples). The main clinical form of dermatophytosis in the sample was tinea unguium ( $42.61 \%)$, followed by tinea pedis $(30.86 \%)$, tinea inguinalis $(11.65 \%)$, tinea corporis $(8.04 \%)$, and tinea manus $(4.76 \%)$. Tinea capitis et faciei $(2.08 \%)$ was more common among children and adolescents.

Conclusion: The assessment of data has showed the predominance of tinea unguium among adult patients, tinea capitis et faciei among children, and the prevalent aetiological role of Trichophyton rubrum in fungal skin infections; findings that are in agreement with recent European studies.
\end{abstract}

Key words: retrospective study, epidemiology, dermatophytes, tinea

Address for correspondence: Z. Baranová, Department of Dermatovenerology, Faculty of Medicine, Pavol Jozef Šafárik University in Košice and Louis Pasteur University Hospital, Trieda SNP 1, 04011 Košice, Slovak Republic. E-mail: zuzana.baranova@upjs.sk

https://doi.org/10.21101/cejph.a5279

\section{INTRODUCTION}

Incidences of fungal infections are increasing throughout the world as a result of the use of broad-spectrum antibiotics, immunosuppressive therapies, cytotoxic agents, chemotherapies and new medical therapeutic methods such as biologics. Other risk groups suffering from fungal infections are most notably in the aged population with increased prevalence of chronic diseases. Socioeconomic status, a warm and humid environment, lifestyle, presence of pets, age and personal hygiene of a patient are also important factors predisposing to dermatophyte infections. Fungal infection risks are highly dependent on a combination of host immune competency and specific exposures of people both within the health care system and their communities (1).

Fungal infection - dermatophytosis - is an infection of the skin, nails and hair caused by dermatophytes. The dermatophytes can use cutaneous keratin as a nutrient because of their keratinophilic and keratinolytic properties. Three ecological groups of dermatophyte species are known: anthropophilic, zoophilic and geophilic. Anthropophilic species are confined as a host and spread from human to human. The zoophilic species' usual hosts are animals, and can spread from animals to human. Geophilic species can spread from soil to humans, but are only occasionally pathogenic for animals or man.

The clinical features of dermatophyte infections result from a combination of keratin destruction and an inflammatory host response. The wide variation in clinical presentation depends on the following factors: species and probably strain of fungus, the size of inoculum, site of body infected, and immune status of host. Anthropophilic species typically produce mild but chronic lesions. Zoophilic and geophilic species tend to produce highly inflammatory reactions in humans (2).

The dermatophytes embrace the three genera Epidermophyton (E.), Trichophyton (T.) and Microsporum (M.). Within those genera are the major anthropophilic species T. rubrum, T. interdigitale. T. tonsurans, T. violaceum, M. audouinii, and E. floccosum; the zoophilic species M. canis, T. verrusocum, T. mentagrophytes, T. equinum, M. nanum, M. disortum, and M. persicolor; and the geophilic species T. terrestre, M. gypseum, and M. fulvum.

The dermatophytosis is usually called the "tinea“ and classified according to the body part involved (scalp dermatophytosis is known as tinea capitis, face dermatophytosis as tinea faciei, 
body dermatophytosis as tinea corporis, pedis dermatophytosis as tinea pedis, etc.).

Sometimes, the lesion caused by dermatophytes is known as "ringworm" because of the anatomical shape.

Dermatophytosis diagnosis is made by direct mycological examination with $10-20 \%$ potassium hydroxide $(\mathrm{KOH})$ of biological material obtained from suspected lesions and by cultivation. Molecular methods such as polymerase chain reactions can sometimes be used.

\section{MATERIALS AND METHODS}

The aim of this work was to retrospectively analyse the epidemiological and mycological profile of a patient population with presumed dermarophyte diagnosis of fungal infections.

During a three-year period (from January 2014 to December 2016) a total of 11,989 clinical samples with lesions of the skin, nails and hair were collected from patients with suspected fungal infections. Samples were obtained from dermatological inpatients and outpatients departments in eastern Slovakia. All samples were investigated at the Mycological Laboratory of the Department of Medical and Clinical Microbiology, University Hospital in Košice. The study comprised all samples of patients with suspected diagnosis of skin fungal infection, regardless of causative species, site of infection, gender, age, or prior therapy.

All samples were processed by direct microscopic examination using $20 \%$ potassium hydroxide $(\mathrm{KOH})$ with Parker ink for the visualization of fungal elements (hyphae and spores). However, microscopy provides no information about the identity of species. Therefore, samples were inoculated onto slants of Sabouraud's dextrose agar (SDA) with chloramphenicol and BD Mycosel Agar (Sabouraud's dextrose agar containing cycloheximide and chloramphenicol). Cultures were incubated for three to four weeks at $25{ }^{\circ} \mathrm{C}$. Samples were identified as negative if there was no fungal growth after four weeks of incubation. The dermatophyte was identified on the basis of macroscopic characteristics such as growth rate, nature of growth, macroscopic appearance, surface texture, colour of colonies, pigmentation on reverse and microscopic morphology (characteristic arrangement, size, shape and type of spores produced, presence of macroconidia, production of chlamydospores and some special characteristics).

\section{RESULTS}

In our study, a total of 11,989 samples were examined over a three year period for suspected cases of skin fungal infection. Only positive cultivations of dermatophytes were evaluated, which is the gold standard for fungal infection diagnosis. Of these 11,989 clinical samples 4,584 (38.24\%) proved positive for micromycetes (direct microscopic examination and/or culture) and 7,405 (61.76\%) were completely negative (direct microscopic examination and cultivation).

The sex and age distributions are shown in Table 1 and 2 . Data analysis showed that 6,684 (55.75\%) were female and 5,305 (44.25\%) male. Study subjects' ages ranged from two to 81 years with a mean age of 42.4 years. Fungal elements (spores and hyphae) were detected in 4,584 (38.24\%) of all clinical samples by
KOH wet mount of which 552 (12.04\%) were Malassezia sp., as the causative agent of Pityriasis versicolor. Further investigation of samples by means of cultivation (Table 3 ) showed the presence of dermatophytes in $45.88 \%$ of cases, (such as Trichophyton sp., Epidermophyton floccosum, and Microsporum sp.,), nondermatophytes in $15.29 \%$ of cases (Aspergillus sp, Onychocola canadensis, Cladosporium sp., Paecilomyces sp., Scedosporium $s p$. ., Scolpulariosis brevicaulis, Fusarium $s p$,) and yeasts in $26.79 \%$ of cases (Candida $s p$ ).

We have evaluated the data on dermatophytoses in correlation with age group (Table 4).

Among all the dermatophyte isolates, T. rubrum (79.08\%) was the most common cause of infection, followed by $T$. interdigitale $(10.60 \%)$, and $T$. tonsurans $(5.14 \%)$, whereas $T$. violaceum, T. mentagrophytes, Microsporum sp. and E. floccosum were the least common. Of the zoophilic species, T. mentagrophytes was the most common pathogen $(3.04 \%)$ followed by M. canis $(0.90 \%)$. Annual occurrences of isolated species showed a constant frequency over the study period (Table 5).

Tinea unguium caused by dermatophytes was the most prevalent form of infection at $42.61 \%(896 / 2,103)$, followed by tinea pedis $(30.86 \%)$, tinea inquinale $(11.65 \%)$, tinea corporis $(8.04 \%)$, tinea manus (4.76\%) and tinea capitis et faciei (2.08\%) (Table 6). Tinea capitis et faciei mostly affected children and adolescents

Table 1. Annual frequency and percentage distributions by gender $(N=11,989)$

\begin{tabular}{|l|c|c|c|}
\hline Year & $\begin{array}{c}\text { Female } \\
\mathbf{n}(\%)\end{array}$ & $\begin{array}{c}\text { Male } \\
\mathbf{n}(\%)\end{array}$ & $\begin{array}{c}\text { Total } \\
\mathbf{n}(\%)\end{array}$ \\
\hline 2014 & $2,094(53.78)$ & $1,800(46.22)$ & $3,894(32.48)$ \\
\hline 2015 & $2,265(54.91)$ & $1,860(45.09)$ & $4,125(34.41)$ \\
\hline 2016 & $2,325(58.56)$ & $1,645(41.44)$ & $3,970(33.11)$ \\
\hline Total & $6,684(55.75)$ & $5,305(44.25)$ & $11,989(100.00)$ \\
\hline
\end{tabular}

Table 2. Gender distribution according to age group $(N=11,989)$

\begin{tabular}{|c|c|c|c|c|c|c|}
\hline \multirow{2}{*}{$\begin{array}{l}\text { Age } \\
\text { group }\end{array}$} & \multicolumn{2}{|c|}{$0-20$ years } & \multicolumn{2}{|c|}{$21-60$ years } & \multicolumn{2}{|c|}{$\geq 61$ years } \\
\hline & Female & Male & Female & Male & Female & Male \\
\hline 2014 & 32 & 40 & 1,196 & 1,039 & 866 & 721 \\
\hline Total & \multicolumn{2}{|c|}{72} & \multicolumn{2}{|c|}{2,235} & \multicolumn{2}{|c|}{1,587} \\
\hline 2015 & 48 & 59 & 1,525 & 1,288 & 692 & 513 \\
\hline Total & \multicolumn{2}{|c|}{107} & \multicolumn{2}{|c|}{2,813} & \multicolumn{2}{|c|}{1,205} \\
\hline 2016 & 46 & 52 & 1,594 & 1,141 & 685 & 452 \\
\hline Total & \multicolumn{2}{|c|}{98} & \multicolumn{2}{|c|}{2,735} & \multicolumn{2}{|c|}{1,137} \\
\hline
\end{tabular}

Table 3. Annual distribution of all micromycetes $(N=4,584)$

\begin{tabular}{|l|c|c|c|c|c|}
\hline Years & $\begin{array}{c}\text { Dermato- } \\
\text { phytes }\end{array}$ & $\begin{array}{c}\text { Nonderma- } \\
\text { tophytes }\end{array}$ & Yeasts & $\begin{array}{c}\text { Malassezia } \\
\text { sp. }\end{array}$ & Total \\
\hline 2014 & 731 & 195 & 472 & 223 & 1,621 \\
\hline 2015 & 736 & 295 & 396 & 189 & 1,616 \\
\hline 2016 & 636 & 211 & 360 & 140 & 1,347 \\
\hline Total & 2,103 & 701 & 1,228 & 552 & 4,584 \\
$\mathrm{n}(\%)$ & $(45.88)$ & $(15.29)$ & $(26.79)$ & $(12.40)$ & $(100.00)$ \\
\hline
\end{tabular}


Table 4. Frequency of clinical presentation of dermatophytoses according to age group $(N=2,103)$

\begin{tabular}{|l|c|c|c|c|c|}
\hline $\begin{array}{l}\text { Clinical } \\
\text { manifestations }\end{array}$ & $\begin{array}{c}\mathbf{0 - 2 0} \\
\text { years }\end{array}$ & $\begin{array}{c}\mathbf{2 1 - 6 0} \\
\text { years }\end{array}$ & $\begin{array}{c}\mathbf{\geq 6 1} \\
\text { years }\end{array}$ & Total & $\%$ \\
\hline Tinea capitis et faciei & 29 & 15 & 0 & 44 & 2.08 \\
\hline Tinea corporis & 28 & 123 & 18 & 169 & 8.04 \\
\hline Tinea manus & 8 & 59 & 33 & 100 & 4.76 \\
\hline Tinea inguinalis & 0 & 118 & 127 & 245 & 11.65 \\
\hline Tinea pedis & 21 & 320 & 308 & 649 & 30.86 \\
\hline Tinea unguium & 4 & 556 & 336 & 896 & 42.61 \\
\hline Total $\mathrm{n}(\%)$ & 90 & 1,191 & 822 & 2,103 & 100.00 \\
\hline
\end{tabular}

Table 5. Annual prevalence of dermatophytes $(N=2,103)$

\begin{tabular}{|l|c|c|c|c|c|}
\hline Dermatophytes & $\mathbf{2 0 1 4}$ & $\mathbf{2 0 1 5}$ & $\mathbf{2 0 1 6}$ & Total & \% \\
\hline T. rubrum & 579 & 584 & 500 & 1,663 & 79.08 \\
\hline T. interdigitale & 67 & 80 & 76 & 223 & 10.60 \\
\hline T. mentagrophytes & 34 & 17 & 13 & 64 & 3.04 \\
\hline T. tonsurans & 39 & 38 & 31 & 108 & 5.14 \\
\hline T. violaceum & 2 & 0 & 1 & 3 & 0.14 \\
\hline T. verrusocum & 1 & 0 & 0 & 1 & 0.05 \\
\hline M. audouinii & 1 & 5 & 3 & 9 & 0.43 \\
\hline M. canis & 5 & 7 & 7 & 19 & 0.90 \\
\hline M. gypseum & 3 & 4 & 3 & 10 & 0.48 \\
\hline Epidermophyton & 0 & 1 & 2 & 3 & 0.14 \\
\hline Total & 731 & 736 & 636 & 2,103 & 100.00 \\
\hline
\end{tabular}

under 20 years of age, while the other forms of tinea, mainly tinea pedis et unguium, were more frequent in adulthood. A significant proportion of patients had onychomycosis and tinea pedis in the adult age range $(p<0.001)$, whereas tinea capitis was more common among children and adolescents $(\mathrm{p}=0.010)$.

Mainly zoophilic species such as T. mentagrophytes, M. canis and antropophilic M. audouinii were associated with tinea capitis in the under 20 years age group. T. rubrum was the predominant species isolated from adults with tinea unguium $(37.95 \%-798 / 2,103)$ and tinea pedis $(27.15 \%-571 / 2,103)$. T. tonsurans was the predominant species isolated (5.13\%) mostly with tinea corporis, while other forms of tinea were less frequent (Table 6).

\section{DISCUSSION}

Dermatophytoses are a worldwide problem. The increasing prevalence of fungal infections may be attributed to an ageing population and the use of immunosuppressive therapies, cytotoxic agents, chemotherapies, and biologic drugs. Other factors may be related to lifestyle, occupation or immune status. The prevalence of fungal infections varies according to geographical location, environmental conditions and cultural and socio-economic factors. The incidence of superficial fungal infections in the global human population is assumed to be 20 to $25 \%$ (3).

In the period between 2014 to 2016, 11,989 samples were obtained from skin, hair and nails and examined in a mycological laboratory. Direct microscopic investigation and/or cultivation gave positive results in $38.24 \%(4.584 / 11,989)$ of samples, while $61.76 \%$ of samples were completely negative. Dermatophytes were detected in $45.88 \%(2,103 / 4,584)$ of all positive samples, yeasts in $26,79 \%(1,228 / 4,584)$, nondermatophytes in $15.29 \%$ $(701 / 4,584)$ and Malassezia sp in $12.04 \%(552 / 4,584)$ of cases.

Berenji et al. (4) found that among 3,804 patients with cutaneous and superficial fungal infections, 2,212 (58.1\%) were positive for Malassezia infections. Furthermore, 1,257 (33.1\%) cases of dermatophytes, 258 (6.8\%) cases of Candida infections, 62 (1.6\%) cases of aspergillosis and $15(0.4 \%)$ cases of other fungal infections were identified. Miklic et al. (5) reported the frequency of superficial fungal infections based on aetiological agents isolated during a ten-year period in Zagreb, Croatia. The results showed that dermatophytes were responsible for $63 \%$ of all superficial fungal diseases, followed by yeasts (36\%) and moulds (1\%).

Malassezia genus yeast belongs to normal skin microflora and is the causative agent of pityriasis versicolor (PV). Clinical diagnosis is simply confirmed by direct microscopical examination with $\mathrm{KOH}$ and demonstration of pseudohyphae and blastoconidia in the typical "spaghetti and meatballs" pattern. Cultivation is not necessary for routine diagnosis. Prevalence is high in regions with hot and humid climates with over $15 \%$ of the population

Table 6. Causative agents of dermatophytosis in correlation with clinical forms

\begin{tabular}{|l|c|c|c|c|c|c|c|}
\hline & $\begin{array}{c}\text { Tinea capitis, } \\
\text { faciei }\end{array}$ & Tinea corporis & Tinea manus & $\begin{array}{c}\text { Tinea } \\
\text { inguinalis }\end{array}$ & Tinea pedis & Tinea unguium & Total $\mathbf{n}(\%)$ \\
\hline T. rubrum & 0 & 45 & 62 & 187 & 571 & 798 & $1,663(79.08)$ \\
\hline T. interdigitale & 0 & 4 & 2 & 54 & 69 & 94 & $223(10.60)$ \\
\hline T. mentagrophytes & 14 & 23 & 22 & 0 & 5 & 0 & $64(3.04)$ \\
\hline T. tonsurans & 5 & 85 & 8 & 2 & 4 & 4 & $108(5.13)$ \\
\hline T. violaceum & 0 & 2 & 1 & 0 & 0 & 0 & $3(0.14)$ \\
\hline T. verrusocum & 0 & 0 & 1 & 0 & 0 & 0 & $1(0.05)$ \\
\hline M. audouinii & 9 & 0 & 0 & 0 & 0 & 0 & $9(0.43)$ \\
\hline M. canis & 12 & 7 & 0 & 0 & 0 & 0 & $19(0.90)$ \\
\hline M. gypseum & 4 & 2 & 4 & 0 & 0 & 0 & $10(0.48)$ \\
\hline Epidermophyton & 0 & 1 & 0 & 2 & 0 & 0 & $3(0.14)$ \\
\hline Total $n$ (\%) & $44(2.08)$ & $169(8.04)$ & $100(4.76)$ & $245(11.65)$ & $649(30.86)$ & $896(42.61)$ & $2,103(100.00)$ \\
\hline
\end{tabular}


potentially affected (6). In Italy, the prevalence of this superficial yeast infection was $2.1 \%$ among 1,024 young Italian sailors (7). We observed a higher incidence of Pityriasis versicolor. This may be explained by the characteristics of our patient population because we have evaluated samples of patients with presumed skin fungal infection.

Of the 2,103 dermatophytes isolates in the present study, $79.08 \%$ was accounted for by $T$. rubrum, followed by $T$. interdigitale (10.60\%), and T. tonsurans (5.13\%). Microsporum sp, Epidermophyton floccosum, T. violaceum, and T. mentagrophytes were rare as causative agents. Tinea ungium predominated in our study (42.61\%), followed by tinea pedis (30.86\%) and tinea inguinalis $(11.65 \%)$. The most common sites of involvement were nails and feet in adults, and scalps in children, which is consistent with the literature. Other recent epidemiological surveys of skin fungal infections have confirmed the increased prevalence of tinea unguium, as well as the predominant etiological role of T. rubrum (8-12).

In a survey of over 96,000 patients in 20 European countries (known as the Achilles Project), onychomycosis was diagnosed in $29.6 \%$ of the population. In over $70 \%$ of diagnoses confirmed by cultivation dermatophytes were the fungi causing infection (13).

According to Vena et al. (14), the most prevalent clinical form was tinea ungium (39.2\% of total dermatophytoses) followed by tinea corporis $(23 \%)$, tinea pedis $(20.5 \%)$, and tinea cruris $(8 \%)$. T. rubrum was the most prevalent causative agent, implicated in $64 \%$ of total cases.

In Sweden (15), onychomycosis had the highest overall prevalence of $14.1 \%$, followed by tinea pedis (4.4\%). Trichophyton rubrum was the predominant pathogen isolated from these cases (83.2\%), followed by $T$. mentagrophytes $(7.4 \%)$.

According to the retrospective analysis from 2006 to 2009 by Simonnet et al. (16), the most prevalent clinical form was onychomycosis ( $28.2 \%$ of total dermatophytoses) and T. rubrum was the most common dermatophyte recovered from cases of onychomycosis $(67.4 \%)$, tinea pedis $(70.6 \%)$ and tinea corporis $(52.4 \%)$.

Our data showed that tinea capitis and tinea faciei were dominant clinical types in the $0-20$ years age group $(65.91 \%, 29 / 44$ cases). These infections were not observed in adults aged over 61 years. Aetiological agents identified with infections were T. mentagrophytes $(31.82 \%, 14 / 44$ cases $)$, M. canis $(27.27 \%$, $12 / 44$ cases), M. audouinii (20.45\%. 9/44 cases), T. tonsurans (11.36\%, 5/44 cases) and M. gypseum 9.09\% (4/44 cases). Increased anthropophilic scalp infections (caused by T. tonsurans. T. violaceum, M. audouinii) are reported in western and northern Europe, while zoophilic Microsporum canis remains the predominant organism with the highest incidence in Central and Southern Europe (17-21).

\section{CONCLUSION}

We conclude that the overall epidemiological and clinicomycological profile of fungal skin infections in eastern Slovakia is in agreement with most published studies.

The assessment of data has showed the predominance of tinea unguium among dermatophytosis and the prevalent aetiological role of T. rubrum.

\section{Acknowledgement}

This work was supported by Grants VEGA No. 1/0198/13, 1/0011/14 of the Ministry of Education, Science, Research and Sport of the Slovak Republic.

\section{Conflict of Interests}

None declared

\section{REFERENCES}

1. Hospenthal DR, Rinaldi MG, editors. Diagnosis and treatment of fungal infections. 2nd ed. Cham: Springer International Publishing Switzerland; 2015.

2. Hay RJ, Moore MK. Mycology. In: Burns T, et al., editors. Rook's textbook of dermatology. 7th ed. Oxford, England: Blackwell; 2004. p. 1405-1506.

3. Havlickova B, Czaika VA, Friedrich M. Epidemiological trends in skin mycoses worldwide. Mycoses. 2008;51 Suppl 4:2-15.

4. Berenji F, Mahdavi Sivaki M, Sadabadi F, Andalib Aliabadi Z, Ganjbakhsh M, Salehi M. A retrospective study of cutaneous fungal infections in patients referred to Imam Reza Hospital of Mashhad, Iran during 2000-2011. Curr Med Mycol. 2016;2(1):20-3.

5. Miklić P, Skerlev M, Budimčić D, Lipozenčić J. The frequency of superficial mycoses according to agents isolated during a ten-year period (1999-2008) in Zagreb area, Croatia. Acta Dermatovenerol Croat. 2010;18(2):92-8.

6. Boekhout T, Guého-Kellermann E, Mayser P, Velegraki A, editors. Malassezia and the skin: science and clinical practice. Heidelberg: Springer-Verlag; 2010 .

7. Ingordo V, Naldi L, Colecchia B, Licci N. Prevalence of pityriasis versicolor in young Italian sailors. Br J Dermatol. 2003;149(6):1270-2.

8. Maraki S, Mavromanolaki VE. Epidemiology of dermatophytoses in Crete, Greece: a 5-year Survey. Med Mycol J. 2016;57(4):69-75.

9. Svejgaard EL, Nilsson J. Onychomycosis in Denmark: prevalence of fungal infection in general practice, Mycoses. 2004;47(3-4):131-5.

10. Hay R. Literature review. Onychomycosis. J Eur Acad Dermatol Venereol. 2005;19 Suppl 1:1-7.

11. Ameen M. Epidemiology of superficial fungal infections. Clin Dermatol. 2010;28(2):97-201.

12. Nenoff $P$, Hanselmayer GG, Tietz HJ. Fungal nail infections - an update Part 1: Prevalence, epidemiology, predisposing conditions, and differential diagnosis. Hautarzt. 2012;63(1):30-8.

13. Roseeuw D. Achilles foot screening project: Preliminary results of patients screened by dermatologists. J Eur Acad Dermatol Venereol. 1999;12 Suppl 1:S6-9.

14. Vena GA, Chieco P, Posa F, Garofalo A, Bosco A, Cassano N. Epidemiology of dermatophytoses: retrospective analysis from 2005 to 2010 and comparison with previous data from 1975. New Microbiol. 2012;35(2):207-13.

15. Drakensjö IT, Chryssanthou E. Epidemiology of dermatophyte infections in Stockholm, Sweden: a retrospective study from 2005-2009. Med Mycol. 2011;49(5):484-8.

16. Simonnet C, Berger F, Gantier JC. Epidemiology of superficial fungal diseases in French Guiana: a three-year retrospective analysis. Med Mycol. 2011;49(6):608-11.

17. Hay RJ, Robles W, Midgley G, Moore MK; European Confederation of Medical Mycology Working Party on Tinea Capitis. Tinea capitis in Europe: new perspective on an old problem. J Eur Acad Dermatol Venereol. 2001;15(3):229-33.

18. Nicola A, Laura A, Natalia A, Monica P. A 20-year survey of tinea faciei. Mycoses. 2010;53(6):504-8.

19. Ginter-Hanselmayer G, Weger W, Ilkit M, Smolle J. Epidemiology of tinea capitis in Europe: current state and changing patterns. Mycoses. 2007;50 Suppl 2:6-13.

20. Binder B, Lackner HK, Poessl BD, Propst E, Weger W, Smolle J, et al. Prevalence of tinea capitis in Southeastern Austria between 1985 and 2008: up-to-date picture of the current situation. Mycoses. 2011;54(3):243-7.

21. Jankowska-Konsur A, Dylag M, Szepietowski JC. Tinea capitis in southwest Poland. Mycoses. 2009;52(2):193-4.

Received December 6, 2017 Accepted in revised form August 27, 2018 